\title{
CONSTITUINTES QUÍMICOS DA ASCÍDIA Didemnum psammatodes (SLUITER, 1895) COLETADA NA COSTA CEARENSE
}

Renata Takeara, João Luis Callegari Lopes* e Norberto Peporine Lopes

Departamento de Física e Química, Faculdade de Ciências Farmacêuticas de Ribeirão Preto, Universidade de São Paulo, Av. do Café, s/n, 14040-903 Ribeirão Preto - SP, Brasil

Paula Christine Jimenez e Letícia Veras Costa-Lotufo

Departamento de Fisiologia e Farmacologia, Universidade Federal do Ceará, CP 3157, 60430-270 Fortaleza - CE, Brasil

Tito Monteiro da Cruz Lotufo

Departamento de Engenharia de Pesca, Universidade Federal do Ceará, CP 12168, 60455-760 Fortaleza - CE, Brasil

Recebido em 7/7/06; aceito em 9/11/06; publicado na web em 14/5/07

\begin{abstract}
CHEMICAL CONSTITUENTS FROM THE ASCIDIAN Didemnum psammatodes (SLUITER, 1895) COLLECTED ON THE SHORES OF CEARA STATE. Chemical investigation of the methanolic extract of the ascidian Didemnum psammatodes has led to the identification of the nucleosides 2'-deoxyuridine (1), thymidine (2), 2'-deoxyinosine (3) and 2'-deoxyguanosine (4), the steroids cholestanol, cholestanone and stigmasterol in mixture and batyl alcohol plus two analogs, 1-heptadecyloxy-2,3-propanediol and 1-nonadecyloxy-2,3-propanediol in mixture. Their structures were proposed by NMR, MS and comparison with literature data and GC-MS analysis.
\end{abstract}

Keywords: chemical contituents; Didemnum psammatodes; tunicate.

\section{INTRODUÇÃO}

O ambiente marinho é considerado uma rica fonte de compostos com atividades biológicas interessantes, como agentes anti-tumorais, antiinflamatórios, analgésicos, imunomodulatórios, alergênicos e antivirais ${ }^{1}$. As ascídias são invertebrados marinhos produtores de uma gama destes compostos, sendo a maioria deles derivados de aminoácidos ${ }^{2,3}$. Alguns destes derivados e alguns alcalóides de ascídias encontram-se em fase de triagem clínica como agentes anti-tumorais ${ }^{1,4}$.

Didemnidae é a maior família de ascídias em termos de número de espécies e tem provado ser uma grande fonte de compostos naturais com diversidade química e potente propriedade biológica. Várias espécies do gênero Didemnum já foram investigadas quanto à composição química, encontrando-se predominância de alcalóides ${ }^{5,6}$, muitos deles com atividade citotóxica ${ }^{5,7-10}$

Didemnum psammatodes apresenta ampla distribuição geográfica, com registros em praticamente todos os mares tropicais, sendo particularmente abundante no litoral brasileiro ${ }^{11}$. Pouca coisa é conhecida acerca de suas propriedades biológicas. Um estudo preliminar que descreveu a atividade citotóxica do extrato bruto das 10 espécies de ascídias mais abundantes da costa do Ceará mostrou que D. psammatodes possui atividade antimitótica sobre ovos de ouriço do mar $^{12}$. Entretanto, não há relatos na literatura sobre a composição química desta espécie. Sendo assim, o presente trabalho descreve os principais componentes químicos da ascídia D. psammatodes.

\section{PARTE EXPERIMENTAL}

\section{Instrumentação e procedimentos gerais}

Os espectros de $\mathrm{RMN}{ }^{1} \mathrm{H}$ foram obtidos em aparelhos de RMN Brucker DRX-400, operando em $400 \mathrm{MHz}$ para ${ }^{1} \mathrm{H}$ e $100 \mathrm{MHz}$ para ${ }^{13} \mathrm{C}$, respectivamente, com as amostras solubilizadas em $\mathrm{CDCl}_{3}$,

*e-mail: joaoluis@usp.br
DMSO deuterado e $\mathrm{D}_{2} \mathrm{O}$. Os espectros bidimensionais de HMQC e HMBC foram obtidos em aparelho DRX-500.

Os espectros de massas foram obtidos em aparelho da Micromass, modelo Micromass Quattro LC com ionização por electrospray a $45 \mathrm{eV}$.

Para identificação dos esteróides foi utilizado cromatógrafo com fase gasosa acoplado a espectrômetro de massas, marca Shimadzu, modelo QP 2010, com coluna capilar HP-1, com $30 \mathrm{~m}$ x 0,25 mm x $0,25 \mu \mathrm{m}$ de espessura de filme, utilizando-se as seguintes condições: temperatura do injetor: $260{ }^{\circ} \mathrm{C}$; gás de arraste: He; temperatura inicial do forno: $200{ }^{\circ} \mathrm{C}$ por $5 \mathrm{~min}$, velocidade de aquecimento a $5{ }^{\circ} \mathrm{C} / \mathrm{min}$ até $280{ }^{\circ} \mathrm{C}$, permanecendo nessa temperatura por 30 min. O espectro de massas foi obtido por impacto de elétrons a 70 eV. O banco de dados utilizado foi o Wiley Mass Spectral Database.

Separações por CLAE foram realizadas em aparelho Shimadzu LC-6A, com detector de UV-visível modelo SPD-6A, colunas Shimpack ODS-C ${ }_{18} 4,6$ × $250 \mathrm{~mm}$ (analítica) e 20 × $250 \mathrm{~mm}$ (preparativa).

Nas separações por cromatografia em coluna (CC) foram utilizadas colunas de vidro de dimensões variadas e como fase estacionária, sílica gel 60 (70-230 mesh ASTM, Sigma) e Sephadex LH20 (25-100 $\mu$, Pharmacia) como suporte, na proporção média de 1:100 entre amostra:adsorvente para sílica e Sephadex LH-20.

Nas análises por cromatografia em camada delgada comparativa (CCDC) foram utilizadas placas de vidro contendo sílica gel $\mathrm{GF}_{254}$ (Merck) e como reveladores foram utilizadas luzes UV com comprimentos de onda de 254 e 366 nm e nebulização com ácido sulfúrico seguida de aquecimento.

Solventes comerciais foram utilizados para cromatografias em coluna e em camada delgada comparativa, e grau cromatográfico para as purificações em CLAE.

\section{Estudo químico da ascídia Didemnum psammatodes}

Exemplares de $D$. psammatodes (606 g de massa úmida) foram coletados manualmente na zona entre-marés da Praia de Ponta Grossa, Icapuí, estado do Ceará. 
Após a coleta, os animais foram lavados com água do mar para remoção de contaminantes, colocados em metanol e mantidos em freezer até o momento da extração. As amostras foram homogeneizadas em um volume de metanol 3 vezes superior à massa úmida total e o material foi, então, filtrado em papel de filtro e seco em evaporador rotativo, sob pressão reduzida, para remoção do metanol, sendo obtidos $12,15 \mathrm{~g}$ de extrato bruto (cerca de $2 \%$ ).

Foram suspensos $8,41 \mathrm{~g}$ de extrato em metanol. O precipitado insolúvel foi removido por centrifugação. A parte solúvel em metanol $(2,70 \mathrm{~g})$ foi fracionada por cromatografia em coluna de Sephadex LH-20 (MeOH) fornecendo 13 frações. A fração 8 foi posteriormente refracionada por cromatografia em Sephadex LH20, fornecendo 5 frações. A subfração 8.2, segunda fração desta coluna, foi purificada por CLAE (coluna ODS preparativa; solvente: $\mathrm{MeOH} / \mathrm{H}_{2} \mathrm{O}$ 1:9; fluxo: $9 \mathrm{~mL} / \mathrm{min}$; detecção: $250 \mathrm{~nm}$ ), fornecendo $1 \mathrm{mg}$ de 2'-deoxiuridina (1), $2 \mathrm{mg}$ de 2'-deoxiinosina (3) e $1 \mathrm{mg}$ de timidina (2). Na subfração 8.3, terceira da coluna, formou-se precipitado branco, o qual foi separado do sobrenadante por filtração. O precipitado (7 mg) foi identificado como 2'-deoxiguanosina (4). Na fração 9 também formou precipitado branco, o qual foi separado do sobrenadante por filtração. O precipitado (4 mg) foi identificado também como 2'-deoxiguanosina (4).

Uma alíquota do extrato insolúvel em metanol $(2,0 \mathrm{~g})$ foi suspensa em diclorometano, ocorrendo solubilização de $0,218 \mathrm{~g}$, o qual foi submetido à cromatografia em coluna (CC) de sílica gel, utilizando-se como eluentes diclorometano, misturas de diclorometano/MeOH e MeOH em gradiente de polaridade, fornecendo 9 frações. A fração 3 (obtida em diclorometano/MeOH 9:1) foi identificada como uma mistura dos esteróides colestanol, colestanona e estigmasterol (28 mg) por CG-EM, cujos dados são citados abaixo. A fração 4 (obtida em diclorometano/MeOH 9:1) foi purificada por CC de sílica gel utilizando-se como eluentes misturas de hexano/AcOEt e $\mathrm{MeOH}$ em gradiente de polaridade, resultando em álcool batílico e seus análogos 2,3-propanodiol, 1heptadeciloxi e 2,3-propanodiol, 1-nonadeciloxi, obtidos e analisados em mistura $(5 \mathrm{mg})$.
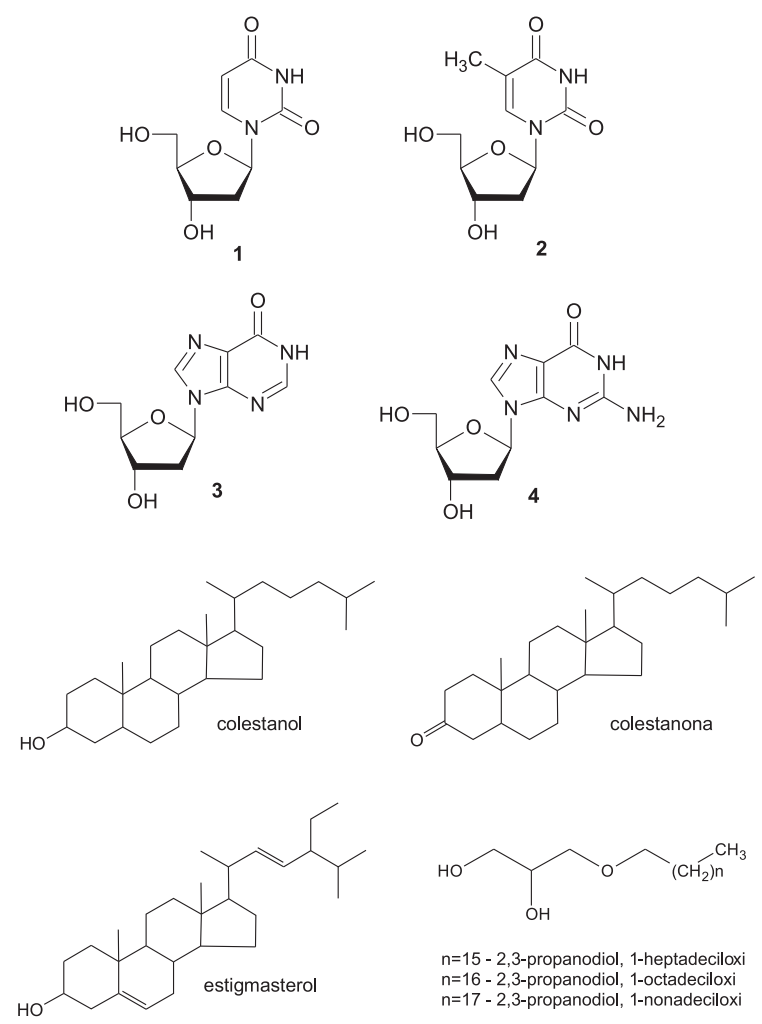

$\mathrm{n}=15-2,3$-propanodiol, 1 -heptadeciloxi

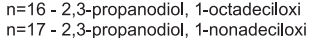

\section{Colestanol}

t.r. $22,437 \mathrm{~min}$; EIMS [ $\mathrm{m} / \mathrm{z}$ (intensidade relativa)]: $388(45 \%$, $\left.\mathrm{M}^{-*}\right), 373$ (38\%), 355 (15\%), 331 (5\%), 262 (17\%), 233 (87\%), 215 (100\%), 165 (41\%), 95 (64\%), 81 (64\%), 55 (61\%).

\section{Colestanona}

t.r. 22,938 $\mathrm{min}$; EIMS [ $\mathrm{m} / \mathrm{z}$ (intensidade relativa)]: $386(35 \%$, $\left.\mathrm{M}^{-\bullet}\right), 371$ (13\%), 231 (100\%), 217 (31\%), 163 (20\%), 95 (38\%), $81(37 \%), 55(45 \%)$.

\section{Estigmasterol}

t.r. $24,708 \mathrm{~min}$; EIMS [m/z (intensidade relativa)]: $412(16 \%$, $\left.\mathrm{M}^{-\bullet}\right), 281$ (10\%), 273 (53\%), 255 (35\%), 161 (40\%), $149(53 \%)$, 137 (52\%), 121 (29\%), 107 (37\%), 97 (50\%), 83 (84\%), 69 (100\%), $55(77 \%)$.

\section{RESULTADOS E DISCUSSÃO}

O fracionamento do extrato metanólico da ascídia $D$. psammatodes resultou no isolamento e identificação dos nucleosídeos 2'-deoxiuridina (1), timidina (2), 2'-deoxiinosina (3) e 2'-deoxiguanosina (4), na identificação da mistura dos esteróides colestanol, colestanona e estigmasterol e da mistura do álcool batílico e seus análogos 2,3-propanodiol, 1-heptadeciloxi e 2,3propanodiol, 1-nonadeciloxi. As estruturas dos nucleosídeos 2'deoxiuridina $^{13,14}$ (1), timidina ${ }^{14,15}$ (2), 2'-deoxiinosina ${ }^{13,14}$ (3) e 2'deoxiguanosina ${ }^{14}(\mathbf{4})$ e do álcool batílico ${ }^{14}$ e seus dois análogos 2,3-propanodiol, 1-heptadeciloxi ${ }^{16}$ e 2,3-propanodiol, 1nonadeciloxi ${ }^{17}$ foram elucidadas com base nas análises dos dados de EM, RMN ${ }^{1} \mathrm{H}$ e ${ }^{13} \mathrm{C}$ e espectros bidimensionais $\operatorname{COSY}{ }^{1} \mathrm{H}-{ }^{1} \mathrm{H}$, HMQC e HMBC, além de comparação com dados publicados anteriormente. Os esteróides foram identificados através de análises por CG-EM, comparando-se os espectros obtidos com os espectros existentes no banco de dados Wiley Mass Spectral Database e com padrões autênticos existentes no laboratório.

Os nucleosídeos observados no presente estudo já foram identificados em diversos organismos marinhos, incluindo ascídias, sendo que timidina já foi encontrada anteriormente no gênero Didemnum $^{18}$. 2'-deoxiuridina, timidina e 2'-deoxiinosina foram isoladas da ascídia Trididemnum cereum ${ }^{13}$, da ascídia Aplidium pantherinum $^{19}$ e da esponja Carteriospongia sp. ${ }^{20}$; 2'-deoxiuridina e timidina foram isoladas da esponja Phakellia mauritiana ${ }^{21}$; timidina foi encontrada na esponja Crambe crambe $^{22}$, na esponja Mycale tenuispiculata ${ }^{23}$, na ascídia Eudistoma sp. $^{24}$ e na ascídia Atriolum robustum ${ }^{25} ; 2^{2}$-deoxiuridina foi isolada da esponja Spongia $\mathrm{sp.}^{26}$ e $2^{\text {' }}$-deoxiguanosina foi encontrada nos briozoários marinhos Cellaria $\mathrm{spp}^{27}$.

Diversas espécies de ascídias foram anteriormente analisadas e mostraram conter misturas com colesterol como componente majoritário ${ }^{28-30}$. O colestanol foi encontrado na túnica da ascídia Phallusia nigra ${ }^{31}$; na ascídia Microcosmus sulcatus ${ }^{28}$; na ascídia Polizoa opuntia ${ }^{30}$ e nas ascídias Styela sp. e Phallusia $\mathrm{sp}^{32}$. A colestanona foi identificada na ascídia Styela sp. $^{32}$ A presença de esteróides vegetais em ascídias, como estigmasterol, pode ser proveniente de fontes dietéticas planctônicas, por serem animais filtradores ${ }^{30,31}$ ou podem ser provenientes de simbiontes ${ }^{30}$.

O álcool batílico e seus análogos também foram encontrados em outras fontes marinhas. O 2,3-propanodiol, 1-heptadeciloxi foi encontrado anteriormente na esponja Haliclona $\mathrm{sp}^{16}$. O álcool batílico também foi isolado da esponja Ulosa ruetzleri ${ }^{33}$; da esponja Tedania ignis $^{34}$, da esponja Haliclona sp. $^{16}$, da esponja Suberites vestigium ${ }^{35}$ e da esponja Mycale mytilorum ${ }^{36}$. O 2,3-propanodiol, 1-nonadeciloxi foi encontrado anteriormente na esponja Suberites vestigium ${ }^{35}$. 
Assim, as substâncias encontradas não são inéditas e estão coerentes com o perfil químico do gênero Didemnum, sendo este o primeiro estudo sobre a composição química da espécie $D$. psammatodes.

\section{AGRADECIMENTOS}

À FAPESP pela bolsa de pós-graduação concedida e ao CNPq, pelos auxílios (Projeto Milênio, processo no 420015/05-1) e bolsas concedidas.

\section{REFERENCIAS}

1. Newman, D. J.; Cragg, G. M.; J. Nat. Prod. 2004, 67, 1216.

2. Davidson, B. S.; Chem. Rev. 1993, 93, 1771.

3. Blunt, J. W.; Copp, B. R.; Munro, M. H. G.; Northcote, P. T.; Prinsep, M. R.; Nat. Prod. Rep. 2006, 23, 26.

4. Rinehart, K. L.; Med. Res. Rev. 2000, $20,1$.

5. Segraves, N. L.; Lopez, S.; Johnson, T. A.; Said, S. A.; Fu, X.; Schmitz, F. J.; Pietraszkiewicz, H.; Valeriote, F. A.; Crews, P.; Tetrahedron Lett. 2003, $44,3471$.

6. Krishnaiah, P.; Reddy, V. L. N.; Venkataramana, G.; Ravinder, K.; Srinivasulu, M.; Raju, T. V.; Ravikumar, K.; Chandrasekar, K.; Ramakrishna, S.; Venkateswarlu, Y.; J. Nat. Prod. 2004, 67, 1168.

7. Carroll, A. R.; Bowden, B. F.; Coll, J. C.; Hockless, D. C. R.; Skelton, B. W.; White, A. H.; Aust. J. Chem. 1994, 47, 61.

8. Rudi, A.; Aknin, M.; Gaydou, E. M.; Kashman, Y.; Tetrahedron 1998, 54, 13203

9. Ham, J.; Kang, H.; Bull. Korean Chem. Soc. 2002, 23, 163.

10. Reddy, S. M.; Srinivasulu, M.; Satyanarayana, N.; Kondapi, A. K.; Venkateswarlu, Y.; Tetrahedron 2005, 61, 9242.

11. Lotufo, T. M. C.; Tese de Doutorado, Universidade de São Paulo, Brasil, 2002.

12. Jimenez, P. C.; Fortier, S. C.; Lotufo, T. M. C.; Pessoa, C.; Moraes, M.E.A.; Moraes, M. O.; Costa-Lotufo, L. V.; J. Exp. Mar. Biol. Ecol. 2003, 287, 93

13. Demattè, N.; Guerriero, A.; De Clauser, R.; De Stanchina, G.; Lafargue, F.; Cuomo, V.; Pietra, F.; Comp. Biochem. Physiol., Part B: Biochem. Mol. Biol. 1985, 81, 479.
14. Pouchert, C. J.; Behnke, J.; The Aldrich library of ${ }^{13} \mathrm{C}$ and ${ }^{1} \mathrm{H} F T$ NMR spectra, Aldrich Chemical Company, 1993, vol. 3.

15. Kitajima, J.; Ishikawa, T.; Tanaka, Y.; Ida, Y.; Chem. Pharm. Bull. 1999, 47, 988.

16. Parameswaran, P. S.; Das, B.; Kamat, S. Y.; Indian J. Chem., Sect. B: Org. Chem. Incl. Med. Chem. 1994, 33, 99.

17. Han, A. R.; Song, J. I.; Jang, D. S.; Min, H. Y.; Lee, S. K.; Seo, E. K.; Arch. Pharmacal. Res. 2005, 28, 290.

18. Mitchell, S. S.; Pomerantz, S. C.; Concepción, G. P.; Ireland, C. M.; J. Nat. Prod. 1996, 59, 1000.

19. Kim, J.; Pordesimo, E. O.; Toth, S. I.; Schmitz, F. J.; Altena, I. V.; J. Nat Prod. 1993, 56, 1813.

20. König, G. M.; Wright, A. D.; Planta Med. 1999, 65, 679

21. Pettit, G. R.; McNulty, J.; Herald, D. L.; Doubek, D. L.; Chapuis, J. C.; Schmidt, J. M.; Tackett, L. P.; Boyd, M. R.; J. Nat. Prod. 1997, 60, 180.

22. Berlinck, R. G. S.; Quim. Nova 1994, 17, 167.

23. Venkatesham, U.; Rao, M. R.; Venkateswarlu, Y.; J. Nat. Prod. 2000, 63, 1318.

24. Schupp, P.; Pochner, T.; Edrada, R.; Ebel, R.; Berg, A.; Wray, V.; Proksch, P.; J. Nat. Prod. 2003, 66, 272.

25. Kehraus, S.; Gorzalka, S.; Hallmen, C.; Iqbal, J.; Müller, C. E.; Wright, A. D.; Wiese, M.; König, G. M.; J. Med. Chem. 2004, 47, 2243.

26. Cao, S.; Gao, Z.; Thomas, S. J.; Hecht, S. M.; Lazo, J. S.; Kingston, D. G. I.; J. Nat. Prod. 2004, 67, 1716.

27. Anthoni, U.; Larsen, C.; Nielsen, P. H.; Christophersen, C.; Lidgren, G.; Comp. Biochem. Physiol. 1989, 92B, 711.

28. Kljajic, Z.; Dogovic, N.; Gasic, M.J.; Comp. Biochem. Physiol., Part B: Biochem. Mol. Biol. 1983, 75, 519.

29. D'Auria, M. V.; Minale, L.; Riccio, R.; Chem. Rev. 1993, 93, 1839.

30. Palermo, J. A.; Brasco, M. F. R.; Hughes, E. A.; Seldes, A. M.; Balzaretti, V. T.; Cabezas, E.; Steroids 1996, 61, 2.

31. Zelnik, R.; Matida, A. K.; Djerassi, C.; Khalil, M. W.; De Freitas, J. C.; An. Acad. Bras. Cienc. 1981, 53, 733.

32. Slantchev, K.; Yalçin, F.; Ersöz, T.; Nechev, J.; Çahs, I.; Stefanov, K.; Popov, S.; Z. Naturforsch., C: J. Biosci. 2002, 57, 534

33. Cardelina II, J. H.; Graden, C. J.; Greer, B. J.; Lipids 1983, 18, 107.

34. Schmitz, F. J.; Vanderah, D. J.; Hollenbeak, K. H.; Enwall, C. E. L.; Gopichand, Y.; Sengupta, P. K.; Hossain, M. B.; Van der Helm, D.; J. Org. Chem. 1983, 48, 3941.

35. Mishra, P. D.; Wahidullah, S.; D' Souza, L. D.; Kamat, S. Y.; Indian J. Chem., Sect B: Org. Chem. Incl. Med. Chem. 1997, 36, 719.

36. Reddy, G. B. S.; Dhananjaya, N.; Bioorg. Med. Chem. 2000, 8, 27. 